\title{
Multiple Symmetrical Lipomatosis in a 67-Year Old African Male: First Case Report within a Resource-Limited Setting
}

\section{Veronique Suttels ${ }^{1^{*}}$, Tertius Venter ${ }^{2}$ and Michelle White ${ }^{3}$}

${ }^{1}$ Clinique Saint-Luc, BP 319 Andabizy, Toliara, Madagascar

${ }^{2}$ Department of surgery, Africa Mercy, Toamasina, Madagascar

${ }^{3}$ Anesthesiologist, Deputy Chief Medical Officer \& Medical Capacity Building Director, Africa Mercy, Toamasina, Madagascar

"Corresponding author: Veronique Suttels, MD, Family Physician, Clinique Saint-Luc, BP 319 Andabizy, Toliara, Madagascar, Tel: 0032473129399; E-mail: Veronique.Suttels@outlook.com

Received date: November 1, 2015, Accepted date: December 9, 2015, Published date: December 13, 2015

Copyright: ( 2015 Suttels, et al. This is an open-access article distributed under the terms of the Creative Commons Attribution License, which permits unrestricted use, distribution and reproduction in any medium, provided the original author and source are credited.

\begin{abstract}
Introduction: Multiple Symmetrical Lipomatosis (MSL) is a progressive disease presenting with symmetrically distributed (large) lipomata over the occiput, head, neck, shoulders and trunk resulting in gross deformity. Over $90 \%$ of cases are associated with chronic alcohol abuse, and associations with metabolic disorders are also common but malignancy is rare. To date there is only one report of MSL in an African-American patient and no reported cases in Africa

Case Presentation: We report the case of a 67-year old African male with a history of gout and chronic heavy drinking presenting with typical features of multiple symmetrical lipomatosis. The lipomata were successfully removed by a visiting surgical mission team.

Discussion: Exact pathogenesis and incidence of MSL remain unclear. Misdiagnosis is common and diagnosis in resource-limited settings should be prompted by the history of alcohol abuse and characteristic presenting features of multiple symmetrical lipomatosis. Challenges of surgical interventions in low-resource settings are discussed.

Conclusion: Clinicians should be aware of the condition and its association with metabolic disorders and rarely malignancy. In resource-limited settings, ethical and cultural factors should be considered before proceeding to surgery.
\end{abstract}

Keywords: Multiple symmetrical lipomatosis; Alcohol abuse; Surgical treatment; Resource-limited setting

\section{List of Abbreviations}

ALP: Alkaline Phosphatase; ALT: Alanine transaminase; AST: Aspartate Transaminase; GGT: Gamma-glutamyl transferase; HDL: High Density Lipoprotein; LDH: Lactate Dehydrogenase; LDL: Low Density Lipoprotein; MSL: Multiple Symmetrical Lipomatosis; TSH: Thyroid Stimulating Hormone

\section{Introduction}

Madelung's disease, Launois-Bensaude syndrome, otherwise known as Multiple Symmetrical Lipomatosis (MSL) is a slow progressing deforming disease presenting with symmetrical, uncapsulated lipomata. Lipomata typically occur in the occipital fossa, head, neck, shoulder and trunk distribution. The lipotama can grow extremely large but distal arms and legs are rarely affected [1,2]. The etiology remains unknown, but over $90 \%$ of cases are associated with chronic alcohol abuse and middle-aged men are the most susceptible [3]. Many other conditions have also been associated with MSL: metabolic syndromes with alterations in glucose tolerance; hypertension and hyperlipidaemia; hyperuricaemia; liver disease; hypothyroidism and elevated parathormone [4-6]. Malignant transformation is reported in the head and neck region [7]. Clinically, the condition causes apnea, obstruction of vessels and nerves, dysphagia, myopathy and reduced head and neck mobility $[8,9]$. Due to the unsightly gross deformities patients often experience stigma and isolation. Treatment aims to control risk factors (e.g. alcohol abstinence) and surgical excision of the lipomata [10]. However, recurrences are described, even in patients who abstain from alcohol [11].

The incidence of MSL is unclear but is reported as highest in the Mediterranean with an estimated incidence of 1 in 25.000 Italian males [12]. There are no case reports from the African continent. We report the first case of MSL diagnosed and treated in a 67-year old African male in Madagascar. Pathogenesis, differential diagnosis and the challenges of treatment and follow-up in a resource limited setting are discussed.

\section{Case Presentation}

The patient travelled $300 \mathrm{~km}$ from his village to a town in Southern Madagascar where the surgical charity, Mercy Ships was known to be conducting a patient selection process to provide free surgical care to those in need. He presented with multiple large, soft and mobile masses in a symmetric distribution along the spine, hypochondrium, 
suprascapular, supraclavicular and occipital regions causing limited neck flexion and extension (Figure 1).

The masses first appeared 3 years ago, initially in the paraspinal region, then ascending to around the face and neck. The patient denied pain, headaches, visual or auditory disturbance, respiratory difficulties or swallowing problems.

He had a past medical history of gout for 5 years and significant alcohol abuse ( 65 centiliters per day of locally produced rum over the course of 50 years), although he reported cessation of alcohol intake since his last consultation for gout one month previously. Neurological, cardiovascular and respiratory examinations were unremarkable.

The patient was transported for further investigations and treatment in the Mercy Ships hospital located $1.268 \mathrm{~km}$ away from the patient selection site.

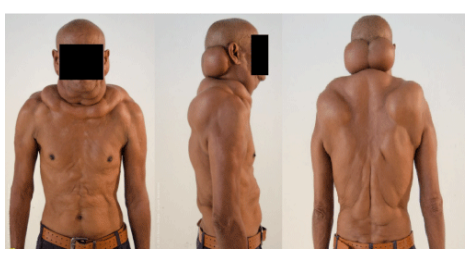

Figure 1: Showing multiple large, soft and mobile masses in a symmetric distribution along the spine, hypochondrium, suprascapular, supraclavicular and occipital regions causing limited neck flexion and extension.

Laboratory results on admission revealed a normal serum glucose (79 mg/dL), elevated total cholesterol of $233 \mathrm{mg} / \mathrm{dL}$ with elevated LDL levels $(152 \mathrm{mg} / \mathrm{dL})$ and normal HDL $(44 \mathrm{mg} / \mathrm{dL})$, elevated triglycerides of $185 \mathrm{mg} / \mathrm{dL}$, strictly normal liver function panel (AST $18 \mathrm{IU} / \mathrm{L}$, ALT $10 \mathrm{IU} / \mathrm{L}$, GGT $41 \mathrm{IU} / \mathrm{L}$, ALP $50 \mathrm{IU} / \mathrm{L}, \mathrm{LDH} 221 \mathrm{IU} / \mathrm{L}$ ) and normal TSH $(1,29 \mathrm{mIU} / \mathrm{L})$. HIV was tested negative. CT scan of the neck and chest demonstrate very extensive symmetric fatty hypertrophic change located intermuscular throughout the neck and spine (Figure 2). No focal masses or calcified deposits can be seen within the hypertrophied fatty areas.

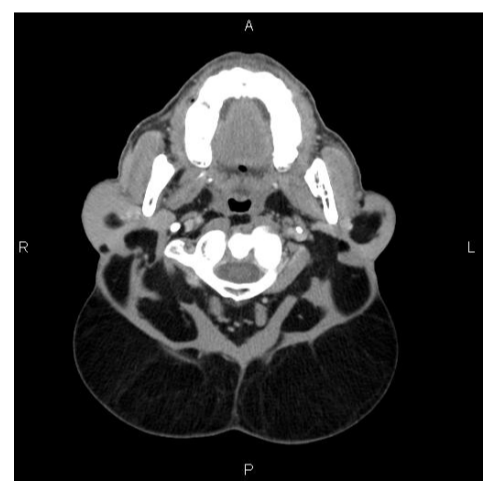

Figure 2: CT scan of the neck.

There was no axillary mediastinal or hilar lymphadenopathy nor intrathoracic fatty hypertrophic change. The patient understood the importance of alcohol abstinence and agreed to a surgical procedure.
Six lipomata were excised in a surgery lasting 5 hours with 1200 milliliters blood loss. Many of the lipomata infiltrated deep into the muscle and surrounding tissues. Cytology results confirmed benign lipomata. Postoperative analgesia was provided with simple oral analgesics and the patient required follow up in outpatients for one month (staying in hotel-style accommodation) and was then discharge home to his village. The postoperative results are shown (Figure 3).

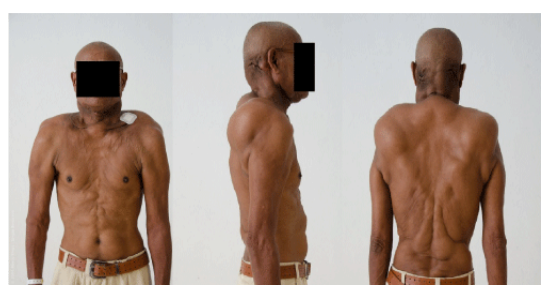

Figure 3: The postoperative results.

Six months later the patient returned for a second operation to excise ten lipomata from the trunk and shoulders. This procedure also lasted 5 hours but had minimal blood loss (300 milliliters) as these lipomata did not infiltrate the muscle as extensively as those excised during the first procedure. Again the patient required one month of outpatient follow up for wound care management and was then discharged home. Post-surgery results up till 5 months were satisfying and the patient was able to restore his village activities.

\section{Discussion}

We present the first case of MSL in Africa. Our case was associated with chronic alcohol abuse but the exact pathophysiological mechanism for this is unclear although mitochondrial disorders (e.g. the m.8344A $>\mathrm{G}$ point mutation) in brown adipocytes have been reported [13,14]. MSL has been described in children but is commonest in middle-aged Caucasian males with a male to female ratio of $15: 1$ and a peak incidence between 40 and 50 years $[15,16]$. There is only one report of an African-American patient and therefore the incidence in Africans is largely unknown [9]. In low resource settings misdiagnosis can easily occur. Differential diagnosis needs to be made with obesity, buffalo hump seen in Cushing's disease and even deep vein thrombosis $[16,17]$. Moreover, since the disease progresses slowly, there is often a delay in diagnosis until extreme deformity becomes apparent. Our patient gave a 3-year history of progressive enlargement of the lipomata, but we suspect a longer prodromal phase with less obvious masses.

Diagnosis remains predominantly clinical with characteristic symmetrical, slow growth of soft, non-tender lipomata in the occipital region, neck, shoulder and trunk. A history of alcohol abuse is an important clue. Since there are no definitive inclusion criteria for clinical diagnosis described in the literature, a histological confirmation showing uncapsulated adipose tissue can be made $[2,16]$. We did not undertake histological diagnosis due to the lack of facilities and difficulty in transporting human tissue from a remote nature of the surgical setting. However, cytology in our case, confirmed benign lipoma. Compression of vital structures (e.g. trachea compression), extent of the lipomata and possible associated malignant processes are best evaluated by MRI or CT [18]. In our case we used CT which showed extensive symmetric superficial and deep intermuscular lipomatosis compatible with MSL. 
Alcohol consumption measured as total adult consumption in litres per capita or pure alcohol per year is very low in Madagascar (2.5 liters). Nonetheless, there may be a higher than expected prevalence of episodic heavy drinking as $10-20 \%$ of male drinkers reports this type of behavior [19]. In our case, the man lived in a rum producing village and his high alcohol consumption was likely a contributing factor to the development of MSL. Therefore in resource-low countries, understanding the local cultural context may be a helpful part of the diagnosis. The lipomata may infiltrate deeply into the muscle and surrounding tissues which can lead to extensive blood loss and a longer duration of surgery than expected. Surgery and anesthesia should therefore be prepared for this especially in low-resource settings. Due to the large incision lines postoperative wound management can also be challenging in resource poor environments. In our case this type of surgical, anaesthesia and nursing care was provided by the nongovernmental organization, Mercy Ships and including follow up in outpatients for wound care management. Surgery in low resource settings always implies additional risks due to numerous challenges such as post-operative infection control and patient follow-up. Therefore the decision to operate should always be made with sufficient consideration of the patient's circumstances and local possibilities [20,21]. In our case, Mercy Ships was able to offer the patient long-term post-operative hospital admission and rehabilitation with satisfying results 5 months post-surgery.

\section{Conclusion}

We report the first case of Multiple Symmetrical Lipomatosis associated with chronic alcohol abuse on the African continent. Diagnosis is predominantly clinical characterized by a symmetrical distribution of large, soft and non-tender lipomata on the background of alcohol abuse. Clinicians should be aware of its association with metabolic disorders and rarely malignant disease. Social stigma is an important aspect of the disease and therefore esthetic surgery can restore a patient to 'normal' life. In a resource-limited setting, we recommend careful consideration of the ethical and cultural context before undertaking surgery.

\section{Consent}

Written informed consent was obtained from the patient for publication of this case report. A copy of the written consent is available for review by the Editor-in-Chief of this journal.

\section{Competing Interests}

The authors declare that they have no competing interests.

\section{Authors' Contributions}

Veronique Suttels was responsible for the literature and primary diagnosis and referral of the patient. Tertius Venter was the patient's surgeon and interpreted further investigations. Michelle White reviewed the manuscript and added valuable comments for the first author.

\section{Acknowledgements}

The authors declare no sources of funding for this case report.

\section{References}

1. Ramos S, Pinheiro S, Diogo C, Cabral L, Cruzeiro C (2010) Madelung disease: a not-so-rare disorder. Ann Plast Surg 64: 122-124.

2. Hadjiev B, Stefanova P, Shipkov C, Uchikov A, Mojallal A (2010) Madelung disease: on the morphologic criteria for diagnosis and treatment. Ann Plast Surg 64: 807-808.

3. Hirose A, Okada Y, Morita E, Tanaka Y (2006) Benign symmetric lipomatosis associated with alcoholism. Internal medicine (Tokyo, Japan) 45: 1001-5.

4. Chuang CC, Cheng YF, Chang HP, Lin CZ (2004) Madelung's disease. Journal of the Chinese Medical Association: JCMA 67: 591-4.

5. Cojocaru DC, Cozma CD, Postolache P (2013) Markers of insulin resistance in a case of Launois-Bensaude syndrome. Revista medicochirurgicala a Societatii de Medici si Naturalisti din Iasi 117: 404-8.

6. Bergmann T, Hosl K, Hahn EG, Harsch IA (2008) Metabolical characteristics in patients with multiple symmetrical lipomatosis. Deutsche medizinische Wochenschrift 133: 2323-2327.

7. Guastella C, Borsi C, Gibelli S, Della Berta LG (2002) Madelung's lipomatosis associated with head and neck malignant neoplasia: a study of 2 cases. Otolaryngol Head Neck Surg 126:191-2.

8. Esteban Julvez L, Perello Aragones S, Aguilar Bargallo X (2013) Sleep apnea-hypopnea syndrome and multiple symmetrical lipomatosis. Archivos de bronconeumologia 49: 86-7.

9. Palacios E, Neitzschman HR, Nguyen J (2014) Madelung disease: multiple symmetric lipomatosis. See comment in PubMed Commons below Ear Nose Throat J 93: 94-96.

10. Bassetto F, Scarpa C, De Stefano F, Busetto L (2014) Surgical treatment of multiple symmetric lipomatosis with ultrasound-assisted liposuction. Annals of plastic surgery 73: 559-62.

11. Brea-Garcia B, Cameselle-Teijeiro J, Couto-Gonzalez I, Taboada-Suarez A, Gonzalez-Alvarez E (2013) Madelung's disease: comorbidities, fatty mass distribution, and response to treatment of 22 patients. Aesthetic plastic surgery 37: 409-16.

12. Guilemany JM, Romero E, Blanch JL (2005) An aesthetic deformity: Madelung's disease. Acta Otolaryngol 125: 328-330.

13. Nisoli E, Regianini L, Briscini L, Bulbarelli A, Busetto L, et al. (2002) Multiple symmetric lipomatosis may be the consequence of defective noradrenergic modulation of proliferation and differentiation of brown fat cells. The Journal of pathology 198: 378-87.

14. Plummer C, Spring PJ, Marotta R, Chin J, Taylor G, et al. (2013) Multiple Symmetrical Lipomatosis--a mitochondrial disorder of brown fat. Mitochondrion 13: 269-276.

15. Shetty C, Avinash KR, Auluck A, Mupparapu M (2007) Multiple symmetric lipomatosis (MSL) of neck in a child (Madelung's disease): report of a rare presentation. Dento maxillo facial radiology 36: 51-4.

16. Mimica M, Pravdic D, Nakas-Icindic E, Karin M, Babic E, et al. (2013) Multiple symmetric lipomatosis: a diagnostic dilemma. Case Rep Med 2013: 836903 .

17. Tai CJ, Huang CT, Voon WC, Lee KT (2014) Madelung's disease mimicking deep vein thrombosis: an unusual case. Int J Cardiol 172 e74-75.

18. Borriello M, Lucidi A, Carbone A, Iannone V, Ferrandina G (2012) Malignant transformation of Madelung's disease in a patient with a coincidental diagnosis of breast cancer: a case report. Diagnostic pathology 7: 116.

19. Global status report on alcohol and health. Geneva: World Health Organization, 2011.

20. Teicher CL, Alberti K, Porten K, Elder G, Baron E, et al. (2014) Medecins sans frontieres experience in orthopedic surgery in postearthquake Haiti in 2010. Prehospital and disaster medicine 29:21-6.

21. Vivekanantham S, Ravindran RP, Shanmugarajah K, Maruthappu M, Shalhoub J (2014) Surgical safety checklists in developing countries. International journal of surgery 12: 2-6. 